\title{
EUCLIDEAN METRIC AND FLAT METRIC OUTSIDE A COMPACT SET
}

\author{
NOBUHIRO INNAMI \\ (Communicated by Jonathan M. Rosenberg)
}

\begin{abstract}
We investigate to what extent there is the gap between Euclidean metrics and flat metrics outside a compact set in complete Riemannian planes.
\end{abstract}

\section{INTRODUCTION}

Let $g$ be a complete Riemannian metric on $\mathbf{R}^{n}$ and let $g_{0}$ be the Euclidean metric. We say that $\left(\mathbf{R}^{n}, g\right)$ is Euclidean outside a compact set if $g-g_{0}$ has compact support. L. Green and R. Gulliver [4] have proved that a plane which is Euclidean outside a compact set is Euclidean if there is no conjugate point. In the present note we discuss the theorem from the viewpoint of the gap-phenomenon shown by $\mathrm{R}$. Greene and $\mathrm{H}$. Wu [5]. They have shown in the proof of Theorem 1 of [5] that $\left(\mathbf{R}^{n}, g\right), n \geq 3$, is Euclidean outside a compact set if it is flat outside some compact set. Namely, the metric $g$ is a modification of Euclidean metric just on a compact set. This phenomenon is not true without additional condition if $n=2$. We prove the following.

Theorem. Let $M$ be a Riemannian plane which is flat outside a compact set $K$. If the total curvature of $M$ is zero and all geodesics in $M-K$ are minimizing, then $M$ is Euclidean outside some compact set.

Since all geodesics are minimizing in a simply connected Riemannian manifold without conjugate points, we have the following from the theorem of $L$. Green and R. Gulliver.

Corollary. Let $M$ be a Riemannian plane without conjugate points. If the Gaussian curvature is zero outside a compact set and the total curvature of $M$ is zero, then $M$ is Euclidean.

The higher dimensional case for Corollary was treated in [7] by a different method. We first give examples which show that the assumption of the Theorem is necessary. Next we prove the Theorem.

Received by the editors May 8, 1987 and, in revised form, July 11, 1988.

1980 Mathematics Subject Classification (1985 Revision). Primary 53C20, 53C22. 
1. Example. (A) (See [4], p. 44). Let $g=d r^{2}+F(r)^{2} d \theta^{2}$ in polar coordinates, where $F(r)=2 r-(1+\exp (2 r /(r-1))) / 2$ for $0 \leq r \leq 1$ and $F(r)=2 r-1 / 2$ for $r \geq 1$. The Gaussian curvature of $\left(\mathbf{R}^{2}, g\right)$ is nonpositive and flat on $r \geq 1$. This implies that the Theorem is not true without the assumption on the total curvature.

(B) The idea is due to Y. Nasu [8, p. 79, see Figure 2, p. 80]. Let $f$ be a $C^{\infty}$ nonnegative function of compact connected support on $\mathbf{R}$. Let $S_{1}=$ $\left\{(x, y, z) \in \mathbf{R}^{3} ;-\infty<x<\infty, y \leq 0, z=0\right\}, S_{2}=\left\{(x, y, z) \in \mathbf{R}^{3} ; 0 \leq\right.$ $z \leq f(x), x \in \operatorname{Supp}(f), y=0\}$, and $S_{3}=\left\{(x, y, z) \in \mathbf{R}^{3} ;-\infty<x<\infty\right.$, $y \geq 0, z=f(x)\}$. The surface $S^{\prime}=S_{1} \cup S_{2} \cup S_{3}$ is smooth except on the curve bounding $S_{2}$. Smoothing out $S^{\prime}$ we have a smooth surface $S$ such that the Gaussian curvature is zero outside a compact set and the total curvature is zero. This implies that the Theorem is false without the assumption of minimality of geodesics in $M-K$.

(C) A complete Riemannian manifold $M=\left(\mathbf{R}^{2}, g\right)$ of nonnegative (or nonpositive) Gaussian curvature is clearly Euclidean if the total curvature is zero. The same phenomenon is not true in the case "outside a compact set". Namely, there is a Riemannian metric $g$ on $\mathbf{R}^{2}$ such that the total curvature is zero and the Gaussian curvature is nonnegative (or nonpositive) outside a compact set (not identically zero): Let $f$ be a function on $[0, \infty)$ such that

(1) $f(r)>0$ on $[0, \infty)$ and $f(r)=1$ on $[0, a]$ for some $a>0$.

(2) $f(r) \geq 1$ (or $f(r) \leq 1$ ) on $[b, \infty)$ for some $b>a$ and $f(r) \rightarrow 1$ as $r \rightarrow \infty$.

(3) $f^{\prime}(r) \leq 0$ (or $f^{\prime}(r) \geq 0$ ) on $[b, \infty)$, but not identically zero. Put

$$
F(r)=\int_{0}^{r} f(r) d r
$$

We consider a Riemannian metric on $\mathbf{R}^{2}$ given by

$$
g=d r^{2}+F(r)^{2} d \theta^{2}
$$

in polar coordinates. Then, if $G(r, \theta)$ is the Gaussian curvature at $(r, \theta)$, then

$$
G(r, \theta)=-\frac{F^{\prime \prime}(r)}{F(r)}=-\frac{f^{\prime}(r)}{F(r)} \geq 0 \quad(\text { or } \leq 0)
$$

for $r \geq b$. Thus, $M=\left(\mathbf{R}^{2}, g\right)$ has nonnegative (or nonpositive, resp.) Gaussian curvature outside a compact set. We calculate the total curvature as follows.

$$
\begin{gathered}
\int_{0}^{2 \pi} \int_{0}^{\infty} G(r, \theta) F(r) d r d \theta=\int_{0}^{2 \pi} d \theta \int_{0}^{\infty}-F^{\prime \prime}(r) d r \\
=2 \pi \lim _{R \rightarrow \infty}\left\{-F^{\prime}(R)+F^{\prime}(0)\right\}=0 .
\end{gathered}
$$

\section{Proof of Theorem}

2.1. (see $[1,2,6])$. Let $M$ be a complete noncompact Riemannian manifold and let $L$ be a ray in $M$, i.e., the image of an isometric map $\gamma:[0, \infty) \rightarrow M$. 
Define a function $f_{L}: M \rightarrow \mathbf{R}$ by $f_{L}(\cdot)=\lim _{t \rightarrow \infty}\{d(\cdot, \gamma(t))-t\}$. We call the function $f_{L}$ the Busemann function of $L$. The Busemann functions are continuous on $M$. Suppose a sequence $\left\{p_{n}\right\} \subset M$ converges to a point $p \in M$ and a sequence $\left\{T_{n}\right\}$ of minimizing geodesics connecting $p_{n}$ to $\gamma\left(t_{n}\right)$ converges to a ray $L_{1}$. We call such an $L_{1}$ a co-ray from $p$ to $L$. We say that a co-ray $L_{1}$ to $L$ is maximal if any extension of $L_{1}$ as a geodesic is not a co-ray to $L$. We say that $L_{1}$ is an asymptote to $L$ if the maximal co-ray $L_{1}$ to $L$ is a straight line, i.e., the image of an isometric map $\alpha:(-\infty, \infty) \rightarrow M$. Let $C(L)$ be the set of all origins of maximal co-rays to $L$. It is known that for any $p \notin C(L)$ there exists the unique co-ray $L_{1}$ form $p$ to $L$ and the Busemann function $f_{L}$ is differentiable at $p$ with gradient vector $-\dot{\gamma}_{1}(0)$, where $\gamma_{1}:[0, \infty) \rightarrow M$ is a representation of $L_{1}$. Suppose the co-ray relation is an equivalence relation. Then, $f_{L}-f_{L_{1}}$ is constant on $M$ for any $L$ and $L_{1}$ which are co-rays to each other if $f_{L}$ and $f_{L_{1}}$ are differentiable on $f_{L}^{-1}(-\infty, a]$ for some $a \in \mathbf{R}$. It should be noted that $C(L)=\phi$ is not assumed.

2.2.. (see $[1,3])$. Let $M=\left(\mathbf{R}^{2}, g\right)$ be a complete Riemannian manifold and let $L$ be a ray with representation $\gamma:[0, \infty) \rightarrow M$. Let $\left\{p_{n}\right\}$ be a sequence converging to a point $p \in M$. We denote by $A(q, t)$ the greatest lower bound of all angles of $L$ and the minimizing geodesics from $q \in M$ to $\gamma(t)$ at $\gamma(t)$. Then, there is a sequence $\left\{t_{n}\right\}$ such that $t_{n} \rightarrow \infty$ and $A\left(p_{n}, t_{n}\right) \rightarrow 0$ as $n \rightarrow \infty$. If $M$ has the total curvature and a straight line $L$ in $M$, then the total curvature of each component of $M-L$ is nonpositive.

Let $L_{1}$ be a co-ray from $p$ to a ray $L$ and let $A$ be a minimizing geodesic connecting $p$ and the origin $q$ of $L$. Let $D$ be the domain bounded by $A, L$ and $L_{1}$ with convex angles $\alpha_{1}$ and $\alpha_{2}$ at $p$ and $q$, respectively. If the total curvature $\varepsilon(D)$ exists, then

$$
\varepsilon(D)=\alpha_{1}+\alpha_{2}-\pi
$$

because of the Gauss-Bonnet formula. When all vertices are in infinity, we get the following. Let $L_{1}$ be a straight line containing a co-ray to a straight line $L$ and let $L_{2}$ be a straight line such that it contains a co-ray to $L_{-}$and $L_{2-}$ contains a co-ray to $L_{1-}$, where $L_{-}$is the reversed geodesic of $L$. If $D$ is the domain bounded by $L, L_{1}$ and $L_{2}$ and the total curvature $\varepsilon(D)$ of $D$ exists, then $\varepsilon(D) \leq-\pi$.

2.3. Proof of theorem We assume that $M$ is a manifold as in the Theorem. Let $L$ be a ray in $M-K$ and $f_{L}$ the Busemann function of $L$. Assume that $m_{1}<f_{L}(q)<m_{2}$ for any $q \in K$. Then, all levels $f_{L}^{-1}(a), a<m_{1}$, of $f_{L}$ are geodesics because $f_{L}^{-1}\left(-\infty, m_{1}\right.$ ] is flat and all geodesics in $M-K$ are minimizing, i.e., all metric balls are isometric to ones in the Euclidean plane. The geodesic is not a closed curve, and, in particular, $f_{L}^{-1}\left(-\infty, m_{1}\right]$ is isometric to the closed Euclidean half-plane with boundary $L_{1}$. By the same argument, we know that $f_{L}^{-1}\left(-\infty, m_{1}\right] \cup f_{L_{1+}}^{-1}\left(-\infty, m_{3}\right] \cup f_{L_{1-}}^{-1}\left(-\infty, m_{4}\right]$ are flat 
for some $m_{3}$ and $m_{4}$. Let $L_{2}=f_{L_{1_{+}}}^{-1}\left(m_{3}\right)$ and $L_{3}=f_{L_{1-}}^{-1}\left(m_{4}\right)$. Then, since $f_{L}^{-1}\left(-\infty, m_{1}\right]$ is the Euclidean half-plane, $L_{2}$ and $L_{3}$ are both asymptotes to $L$ with suitable orientation, say $L_{2+}$ and $L_{3+}$.

We now want to prove that $L_{2-}$ is a co-ray to $L_{3-}$. If this is not true, we have two possibilities; (1) all co-rays to $L_{3-}$ from all points of $L_{2-}$ going to infinity intersect $K$, and (2) there is a co-ray $L_{0}$ to $L_{3-}$ from some point $p_{0}$ of $L_{2-}$ such that it does not intersect $K$ and is not a subray of $L_{2-}$. Suppose (1) occurs. Let $\left\{p_{n}\right\} \subset L_{2-}$ be a sequence going to infinity and let $B_{n}$ be a co-ray from $p_{n}$ to $L_{3-}$ for each $n$. Since all $B_{n}$ intersect $K$, there is a subsequence of $\left\{B_{n}\right\}$ converging to a straight line $A$. Then, $A$ is such that $A_{+}$contains a co-ray to $L_{3-}$ and $A_{-}$contains a co-ray to $L_{2-}$. Hence, the total curvature $\varepsilon(D)$ of the domain $D$ bounded by $L_{2}, L_{3}$ and $A$ is less than or equal to $-\pi$ because of 2.2. Since the total curvature of the union $M-D$ of three half-plane is nonpositive, we have that $\varepsilon(M) \leq-\pi$, a contradiction. Suppose (2) occurs. Let $D$ be the domain bounded by $L_{3}, L_{0}$ and the subray of $L_{2+}$ from $p_{0}$. The total curvature $\varepsilon(D)$ of $D$ is $\alpha-\pi<0$, where $\alpha$ is the angle of $D$ at $p_{0}$, because of the Gauss-Bonnet formula. Since $K \subset D$, the total curvature of $M-D$ is zero. Hence, $\varepsilon(M)<0$, a contradiction. Thus, we proved that $L_{2-}$ is a co-ray to $L_{3-}$. In particular, if $m_{5}<f_{L_{2-}}(q)$ for $q \in K$, then $L_{2-}$ and $L_{3-}$ are parallel in the Euclidean half-plane $f_{L_{2-}}^{-1}\left(-\infty, m_{5}\right]$. We assume that the distance between $L_{2-}$ and $L_{3-}$ is $l_{2}$.

In order to prove that the support of $g-g_{0}$ is compact, it suffices to prove that $l_{2}=l_{1}$, where $l_{1}$ is the distance between $L_{2+}$ and $L_{3+}$ in the Euclidean half-plane $f_{L}^{-1}\left(-\infty, m_{1}\right]$. Let $L_{4}$ be a straight line in $F_{L_{2-}}^{-1}\left(-\infty, m_{5}\right]$ which contains a minimal geodesic from $L_{3-}$ to $L_{2-}$. Then, by Euclidean geometry in $f_{L_{1+}}^{-1}\left(-\infty, m_{3}\right], L_{4+}$ is an asymptote to $L_{1+}$, and, moreover, $f_{L_{1+}}-f_{L_{4+}}=$ const. on $M$, because $f_{L_{i+}}, i=1,4$ are differentiable in $f_{L_{1+}}^{-1}\left(-\infty, m_{3}\right]$ and the co-ray relation for $L_{1+}$ is transitive. The same is true for $L_{1-}$ and $L_{4-}$. Suppose for the indirect proof that $l_{2} \neq l_{1}$, say $l_{2}>l_{1}$. Let $p=L_{3} \cap L_{4}$, $f_{L_{4+}}(p)=a$ and $f_{L_{4-}}(p)=b . L_{3}$ is a level of $f_{L_{4-}}$. Since $L_{4}$ is a straight line, $f_{L_{4+}}^{-1}(a)$ cannot have any interior point of $f_{L_{4}-}^{-1}(-\infty, b]$. On the other hand, the point of $L_{1}$ backward on $L_{1+}$ with distance $l_{2}$ from $L_{2} \cap L_{1}$ is contained in both $f_{L_{4+}}^{-1}(a)$ and is an interior point $f_{L_{4-}}^{-1}(-\infty, b]$, a contradiction. The Theorem is proved.

\section{REFERENCES}

1. H. Busemann, The Geometry of geodesics, Academic press, New York, 1955.

2. __ Recent synthetic differential geometry, Springer, Berlin-Heidelberg-New York, 1970.

3. S. Cohn-Vossen, Totalkrümung und geodätische Liniien auf einfach zusammenhängenden, offenen, vollständiegen Flächenstüchen, Mat. Sb. (N.S.) 1 (1936), 139-164.

4. L. W. Green and R. Gulliver, Planes without conjugate points, J. Differential Geom. 22 (1985), 43-47. 
5. R. E. Greene and H. Wu, Gap theorems for noncompact Riemannian manifolds, Duke Math. J. 49 (1982), 731-756.

6. N. Innami, Differentiability of Busemann functions and total excess, Math. Z. 180 (1982), 235-247.

7. _, The n-plane with integral curvature zero and without conjugate points, Proc. Japan Acad. Ser. A 62 (1986), 282-284.

8. Y. Nasu, On asymptotes in a metric space with non-positive curvature, Tôhoku Math. J. 9 (1957), 68-95.

Department of Mathematics, Nagoya Institute of Technology, Gokiso, Showa, NAGOYA, 466, JAPAN

Department of Mathematics, Faculty Of Science, Nigata University, NigGata, 950-21, JAPAN 